\title{
The relationship between prescription of ultrafiltration and intradialytic hypotension in Chinese hemodialysis patients
}

\author{
Fei Deng ${ }^{1,2 \#}$, Wenjia $\mathrm{Di}^{3 \#}$, Yuexian $\mathrm{Ma}^{4}$, Yi $\mathrm{Li}^{1}$, Tianping Liao ${ }^{5}$, Junjie $\mathrm{Mou}^{2}$, Daqing Hong ${ }^{1}$, Wei Wang ${ }^{1}$, \\ Jin Chen ${ }^{1}$, Peidu Jiang ${ }^{6}$, Guisen $\mathrm{Li}^{1}$, Jiayan Mao ${ }^{1}$, Jing Feng ${ }^{7}$ \\ ${ }^{1}$ Department of Nephrology, Sichuan Provincial People's Hospital, University of Electronic Science and Technology of China, Chengdu, China; \\ ${ }^{2}$ Department of Nephrology, Chengdu Jinniu District People's Hospital, Chengdu, China; ${ }^{3}$ Clinical Immunology Translational Medicine Key \\ Laboratory of Sichuan Province \& Organ Transplantation Center, Sichuan Provincial People's Hospital, University of Electronic Science and \\ Technology of China, Chengdu, China; ${ }^{4}$ Department of Nephrology, Chengdu University of Traditional Chinese Medicine, Chengdu, China; \\ ${ }^{5}$ Department of Nephrology, Yilong County People's Hospital, Nanchong, China; ${ }^{6}$ Department of Pharmaceutical, Sichuan Provincial People's \\ Hospital, University of Electronic Science and Technology of China, Chengdu, China; ${ }^{7}$ Department of Traditional Chinese Medicine, Sichuan \\ Provincial People's Hospital, University of Electronic Science and Technology of China, Chengdu, China \\ Contributions: (I) Conception and design: J Mao, J Feng; (II) Administrative support: J Mao, J Feng; (III) Provision of study materials or patients: F \\ Deng, W Di; (IV) Collection and assembly of data: Y Ma, Y Li, T Liao, J Mou, D Hong, W Wang, J Chen, P Jiang, G Li, F Deng, W Di; (V) Data \\ analysis and interpretation: F Deng, W Di; (VI) Manuscript writing: All authors; (VII) Final approval of manuscript: All authors. \\ \#These authors contributed equally to this work. \\ Correspondence to: Jiayan Mao. Department of Nephrology, Sichuan Provincial People's Hospital, University of Electronic Science and Technology of \\ China, Chengdu, China. Email: meijuxin6678784@yeah.net; Jing Feng. Department of Traditional Chinese Medicine, Sichuan Provincial People’s \\ Hospital, University of Electronic Science and Technology of China, Chengdu, China. Email: fengjing_here@126.com.
}

Background: Intradialytic hypotension (IDH) remains the most frequent severe side effect of hemodialysis (HD) and increases patient morbidity and mortality. Excessive ultrafiltration (UF) is considered the leading cause of IDH. This study developed a suitable prescription of UF to reduce the incidences of IDH episodes.

Methods: A retrospective study was performed to analyze 33,224 HD/hemodiafiltration (HDF) treatments in 312 patients. The prescription of UF were determined following the International Society of Peritoneal Dialysis (ISPD) guideline. The Pearson's method was used to study the correlation between relative variables. The receiver operating characteristic (ROC) curve was used to predict the value of the UF/weight ratio $(\mathrm{UF} / \mathrm{Wt}$ ) for IDH in all patients to establish a diagnostic cut-off point. Univariate and multivariate logistic regression analyses were applied to study the risk factors of IDH.

Results: Twelve thousand five hundred and fifty-eight sessions of IDH (38.7\%) were identified, among which 1,224 (3.6\%) were recorded with intervention against IDH. Both the systolic blood pressure (SBP) and mean arterial pressure (MAP) of the hemodialytic patients were positively correlated with the UF quantity and the UF/Wt, but negatively correlated with blood flow. The ROC curve showed that UF/Wt $=0.04$ was the cut-off point for IDH. Age [per 10-year increment, odds ratio $(\mathrm{OR})=1.005,95 \%$ confidence interval (CI): 1.004 to $1.007, \mathrm{P}=0.000$ ], diabetes mellitus ( $\mathrm{OR}=1.209,95 \% \mathrm{CI}: 1.122$ to $1.303, \mathrm{P}=0.000)$, and $\mathrm{UF} / \mathrm{Wt}>0.04(\mathrm{OR}=1.605,95 \% \mathrm{CI}: 1.532$ to $1.682, \mathrm{P}=0.000)$ were all independently associated with higher incidences of IDH.

Conclusions: IDH commonly occurs during HD in Chinese patients. Unchangeable factors such as diabetes and age, and modifiable factors including UF were associated with IDH. A UF/Wt threshold more than 0.04 may be a potential alert for avoiding IDH, especially in the elderly and diabetic patients.

Keywords: Maintenance hemodialysis (MHD); ultrafiltration (UF); intradialytic hypotension (IDH)

Submitted Feb 24, 2021. Accepted for publication May 06, 2021.

doi: 10.21037/apm-21-791

View this article at: http://dx.doi.org/10.21037/apm-21-791 


\section{Introduction}

Chronic kidney disease (CKD) has become a worldwide public health problem. An epidemiological investigation revealed a national prevalence rate of $10.8 \%$ in China (1). It is estimated that more than 1 million patients with endstage renal disease (ESRD) require renal replacement therapy, however, most of these patients have not received a kidney transplant, but instead, rely on maintenance hemodialysis (MHD) for survival. According to the Chinese National Renal Data System, there were 603,581 patients on MHD by the end of 2019 (2). One of the most common complications of MHD is intradialytic hypotension (IDH), which not only impacts the process of hemodialysis (HD), but also affects the patient's quality of life (QoL), arteriovenous fistulation (AVF), deep venous catheterization (VC), hospitalization, and even mortality. The main factors that contribute to IDH have long been discussed and modified in clinic (3). However, the incidences of IDH can range from $20 \%$ to $30 \%$, and an inappropriate prescription of ultrafiltration (UF) is the major cause of IDH (4). Thus, much attention should be given to an appropriate prescription of UF to decrease the risk of IDH in MHD patients.

This single-center study aimed to explore the incidences of IDH, the relationship between the UF prescription and IDH, and other risk factors for IDH. The data of 33,224 dialysis treatment sessions from 312 MHD patients were analyzed from the renal treatment system (RTS) database in our hospital.

We present the following article in accordance with the STROBE reporting checklist (available at http://dx.doi. org/10.21037/apm-21-791).

\section{Methods}

\section{Study design}

Between January 2019 and January 2020, patients aged from 18 to 75 years with HD vintage more than 3 months were selected from the Blood Purification Center in the Sichuan Provincial People's Hospital. The in-center HD frequency was 2-3 times weekly. The following patients were excluded: (I) patients who were pregnant or lactating; (II) patients with planned kidney transplantation within half a year; (III) patients with malignancies, active tuberculosis, severe malnutrition [albumin (ALB) $<26 \mathrm{~g} / \mathrm{L}$ ], severe heart failure [New York Heart Association (NYHA) class III or more], severe anemia [hemoglobin $(\mathrm{Hb})<60 \mathrm{~g} / \mathrm{L}$ ], severe pulmonary diseases (such as chronic obstructive pulmonary disease and pneumosilicosis), or severe infections (such as sepsis); (IV) patients who cannot undergo upper-limb monitoring of blood pressure (BP); (V) patients who cannot have regular HD due to any reason (such as psychiatric or economic reasons, or poor compliance); and (VI) patients with major bleeding.

The study protocol was approved by the ethics committee of Sichuan Provincial People's Hospital. All participants provided written informed consent prior to data collection. All procedures performed in this study involving human participants were in accordance with the Declaration of Helsinki (as revised in 2013).

\section{Dialysis setting}

All patients were dialyzed for 4 hours with the Fresenius (Fresenius medical care, St. Wendel, Germany) 4008S, 4008H, 5008, or 5008S HD machine. Low-flux polysulfone hollow-fiber dialyzers F6 (Fresenius) and P1350 (Minntech) were used for common HD, and Fresenius F60S disposable filters were used for hemodiafiltration (HDF). International Society of Peritoneal Dialysis (ISPD) guideline was followed. Low molecular heparin or heparin-free dialysis (with periodic tube wash with saline) were selected as anticoagulation methods. The composition of the dialysate was as follows: $1.25 \mathrm{mmol} / \mathrm{L}$ calcium; $135-140 \mathrm{mmol} / \mathrm{L}$ sodium; and $0.5 \mathrm{mmol} / \mathrm{L}$ magnesium. The Fresenius two grade reverse osmosis water management system (Germen Raul) was used.

\section{Data collection}

All data were obtained from the electronic medical records from the RTS database in our hospital using a de-identified and anonymized method. Demographic characteristics (age, gender, race, height, and dialysis vintage), comorbidities (hypertension, diabetes, and chronic vascular diseases), physical examinations (pre- and post-dialysis weight, interdialytic weight gain, and BP), blood tests, and details of HD prescription [session length, UF volumes, blood flow, etc.] were collated.

\section{Definitions}

BP was collected before and after each dialysis session, as well as hourly during the session. Pulse pressure and mean arterial pressure (MAP) were calculated. IDH was 
Table 1 Baseline patient characteristics and history of comorbidities

\begin{tabular}{lc}
\hline Characteristic & Value \\
\hline Age $(\mathrm{yr})$ & $61.4 \pm 14.5$ \\
Gender & \\
Male, $\mathrm{n}(\%)$ & $166(53.2)$ \\
Female, $\mathrm{n}(\%)$ & $146(46.7)$ \\
Dialysis vintage (months) & $69.7 \pm 53.3$ \\
DM, n (\%) & $30(9.6)$ \\
CVD, n (\%) & $29(9.3)$
\end{tabular}

DM, diabetes mellitus; CVD, cardiovascular diseases. Coronary heart disease is defined as chronic heart failure with predominating left ventricular hypertrophy.

defined according to the $2002 \mathrm{~K} / \mathrm{DOQI}$ (Kidney Disease Outcomes Quality Initiative) Guidelines with the following modification: a decrease in systolic blood pressure (SBP) $\geq 20 \mathrm{mmHg}$ (or a decrease in $\mathrm{MAP} \geq 10 \mathrm{mmHg}$ ) during HD/ HDF with or without symptoms of hypotension, namely, abdominal discomfort, nausea and vomitus, syncope, etc. or any condition in need of medical intervention.

The prescription of UF was defined as the prescribed UF adjusted by body weight (UF/Wt). Cardiovascular disease was defined as chronic heart failure with predominating left ventricular hypertrophy or diagnosed coronary heart disease.

\section{Statistical analysis}

Continuous data are presented as mean \pm standard deviation (SD) or median and interquartile range (IQR). Categorical variables are presented as proportions. Pearson's method was used to study the correlation between BP changes, UF/Wt, and blood flow. Univariate and multivariate logistic regression analyses were applied to study the risk factors of IDH. The receiver operating characteristic (ROC) curve was used to study the predicted value of UF/Wt for IDH in all patients to establish a diagnostic cut-off point of UF/Wt for IDH.

All analyses were performed with SPSS, version 22.0.0.0 (IMB Corp., New York, USA). A P value less than 0.05 was considered statistically significant.

\section{Results}

\section{Patient characteristics}

This study enrolled 312 HD patients (53.2\% males) with a mean age of $61.4 \pm 14.5$ years and a mean dialysis vintage of 69.7 \pm 53.3 months. Among the $312 \mathrm{HD}$ patients, 30 (9.6\%) presented with diabetes mellitus (DM) and 29 (9.3\%) had cardiovascular diseases (CVD) (Table 1).

\section{The incidence of IDH}

Overall, 33,224 dialysis sessions were reviewed and 12,558 sessions of IDH (38.7\%) were identified. Among the IHD cases, intervention against IHD was only recorded in 1224 $(3.6 \%)$ cases.

\section{The association between BP changes and dialysis-related factors}

Statistical analyses revealed that the decreasing amplitude of SBP was positively correlated with $\mathrm{UF}(\mathrm{R}=0.136, \mathrm{P}<0.05)$ and $\mathrm{UF} / \mathrm{Wt}(\mathrm{R}=0.123, \mathrm{P}<0.05)$, but negatively correlated with blood flow $(\mathrm{R}=-0.019, \mathrm{P}<0.05$; Figure 1).

Similarly, the decreasing amplitude of MAP was positively correlated with $\mathrm{UF}(\mathrm{R}=0.136, \mathrm{P}<0.05)$ and $\mathrm{UF} / \mathrm{Wt}(\mathrm{R}=0.123, \mathrm{P}<0.05)$, but negatively correlated with blood flow $(\mathrm{R}=-0.019, \mathrm{P}<0.05$; Figure 2$)$.

\section{Factors associated with IDH}

The ROC curve analyses revealed a cut-off value of 0.04 for UF/Wt. Univariate logistic regression analyses showed that patient characteristics including age, comorbid DM, and comorbid CVD were possible risk factors for IDH $(\mathrm{P}<0.10)$. Further multivariate logistic regression analyses revealed that age [per 10-year increment, odds ratio $(\mathrm{OR})=1.005$, $95 \%$ confidence interval $(\mathrm{CI}) 1.004$ to $1.007, \mathrm{P}<0.001]$, DM ( $\mathrm{OR}=1.209,95 \% \mathrm{CI}: 1.122$ to $1.303, \mathrm{P}<0.001)$, and $\mathrm{UF} / \mathrm{Wt}$ $>0.04$ ( $\mathrm{OR}=1.605,95 \%$ CI: 1.532 to1.682, $\mathrm{P}<0.0001)$ were independently associated with a higher incidence of IDH (Table 2).

\section{Discussion}

IDH is a common complication during HD and is strongly associated with patient outcomes, including QoL, hospitalization, AVF or VC failure, and CVD-associated mortality (5-9). In this study cohort involving 33,224 dialysis sessions, the incidence of IDH was $38.7 \%$, including both patients in need of intervention and asymptomatic patients. Patients in need of intervention were also included because with increasing dialysis vintage, patients will 

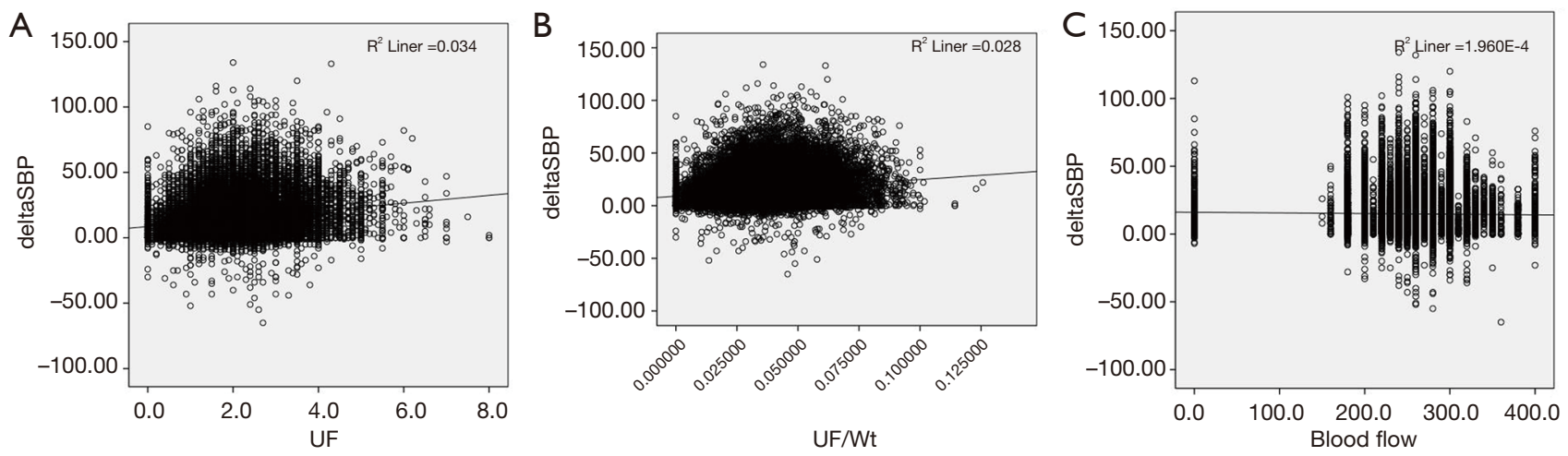

Figure 1 Correlations between UF, UF/Wt, blood flow and SBP changes. A scatter diagram demonstrating the relationships between systolic blood pressure and (A) ultrafiltration, (B) UF/Wt, and (C) blood blow. The decreasing amplitude of SBP was positively correlated with UF $(\mathrm{R}=0.136, \mathrm{P}<0.05)$ and $\mathrm{UF} / \mathrm{Wt}(\mathrm{R}=0.123, \mathrm{P}<0.05)$, but negatively correlated with blood flow $(\mathrm{R}=-0.019, \mathrm{P}<0.05)$. SBP, systolic blood pressure; deltaSBP, SBP before hemodilysis minus SBP after hemodilysis; UF, ultrafiltration; UF/Wt, ultrafiltration weight ratio.
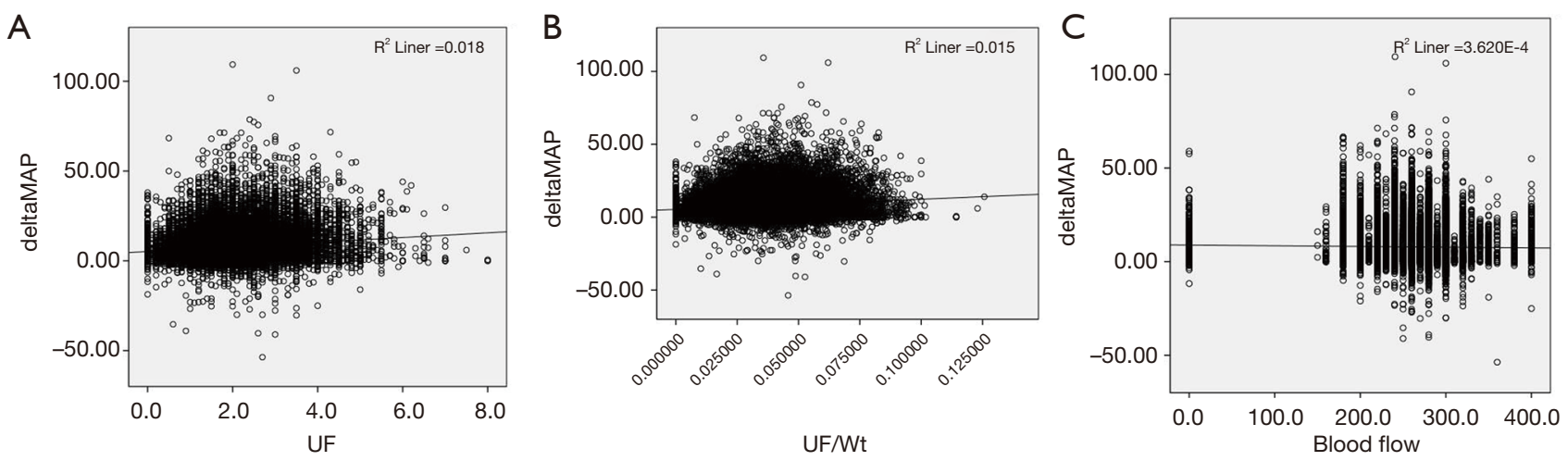

Figure 2 Correlations between UF, UF/Wt, blood flow, and MAP changes. A scatter diagram demonstrating the relationship between mean arterial pressure and (A) ultrafiltration, (B) UF/Wt, and (C) blood flow. The decreasing amplitude of MAP was positively correlated with $\mathrm{UF}(\mathrm{R}=0.136, \mathrm{P}<0.05)$ and $\mathrm{UF} / \mathrm{Wt}(\mathrm{R}=0.123, \mathrm{P}<0.05)$, but negatively correlated with blood flow $(\mathrm{R}=-0.019, \mathrm{P}<0.05)$. MAP, mean arterial pressure; UF, ultrafiltration; deltaMAP, MAP before hemodilysis minus MAP after hemodilysis; UF/Wt, ultrafiltration weight ratio.

Table 2 Multivariate analysis of the main risk factors for IDH

\begin{tabular}{lccc}
\hline Risk factor & OR & $95 \% \mathrm{Cl}$ & $\mathrm{P}$ \\
\hline Age & 1.005 & $1.004-1.007$ & 0.000 \\
DM & 1.209 & $1.122-1.303$ & 0.000 \\
CVD & 1.004 & $0.930-1.085$ & 0.917 \\
UF/Wt $>0.04$ & 1.605 & $1.532-1.682$ & 0.000 \\
\hline
\end{tabular}

Age, comorbid DM, CVD, and UF/Wt (>0.04) were identified as possible risk factors for IDH through single factor analyses with $\mathrm{P}<0.1$. These were further tested through logistic regression analysis and age, DM, and UF/Wt $>0.04$ were all confirmed to be independent risk factors for IDH. CVD, however, was not obviously correlated with IDH and this relationship requires further analysis. IDH, intradialytic hypertension; DM, diabetes mellitus; CVD, cardiovascular diseases; UF/Wt, ultrafiltration/weight ratio; OR, odds ratio; CI, confidence interval. 
become increasingly intolerant to volume removal during dialysis.

Several risk factors have been reported to be associated with IDH, including age, hypertension, DM, lower dry weight, and higher phosphorus levels (10-12). In agreement with these reports, this current study revealed that age (per 10 year-increment), DM, and UF/Wt $>0.04$ were all independently associated with an increased incidence of IDH. Among these risk factors, UF is of significance as it can be altered by medical staff and patients. In some cases, IDH was observed even with a small amount of UF, suggesting that there may be an increase in the UF/Wt. It is generally accepted that a UF/Wt over $6-7 \%$ will cause inadequate effective blood volume which can directly increase the propensity of IDH events. In this study, fluctuations in both SBP and MAP increased with the increase of UF/Wt, attributing to IDH. Analysis using ROC curves demonstrated that $\mathrm{UF} / \mathrm{Wt}=0.04$ was a potential cutoff point for IDH both in patient with and without DM.

To further verify this finding, the potential risk factors of IDH (including age, DM, and CVD) were adjusted. A $\mathrm{UF} / \mathrm{Wt}$ value more than 0.04 in the dialysis prescription was found to be independently associated with IDH. This preliminary cut-off value will be useful as an early alert system allowing patients and medical staff to avoid excessive UF during HD. More precise prediction models using prospectively designed clinical trials will need to be established to further confirm and individualize the clinical application of this finding. Furthermore, medical staff and patients can play an important role in decreasing the incidence of IHD and improving relevant patient outcomes by improved education and methods such as text-message and other electronic-based alert systems.

Certain studies have demonstrated that the rapid removal of sodium, urea, and other osmotically active substances from the intra-vascular compartment, in conjunction with delayed re-equilibration from intracellular compartments, may result in a transient decline in plasma osmolality and intravascular volume depletion secondary to transcellular movement of water $(13,14)$. However, our study found a negative association between blood flow and arterial BP. Similarly, Trivedi et al. demonstrated an increase in systolic and diastolic BP with increasing extracorporeal blood flow rate (EBFR) (15). Meanwhile, a recent study showed no consistent trend in $\mathrm{BP}$ changes induced by a reduction in EBFR, and none of the patients experienced IDH in that study (16). The impact of changes in EBFR on BP during IDH warrants further investigation.
There were some limitations in this study. First, it was a retrospective study, and not all the clinical information could be obtained. The database for HD patients was designed to capture information including patient characteristics, treatment, laboratory findings, and outcomes. Most of the patient clinical information could be collated, and we were able to extrapolate other data relating to treatment and outcomes. Second, the follow-up period was relatively short, and it was not possible to analyze the relationship between UF/Wt and the long-term outcomes. However, this database continuously collects and updates important patient outcomes. This, together with new strategies combining efforts from medical staff (electronic alert, education, etc.) and patients (text-message, education, diet, etc.) will help to develop a more precise prediction model based on the findings of this current study.

In conclusion, IDH is a common complication in dialysis patients, and the associated factors include unchangeable factors such as DM and age, and modifiable factors including UF. A UF/Wt threshold more than 0.04 may be a potential warning to avoid IDH, especially in the elderly and diabetic patients.

\section{Acknowledgments}

Funding: This study was supported by The National Natural Science Foundation of China (Grant No. 81700581), Project of Science and Technology Department of Sichuan Province (Grant No. 2019YFH0069), Chengdu Medical Research Project (Grant No. 2020208), and the Sichuan Provincial People's Hospital Clinical Research and Transformation Fund (2018LY12).

\section{Footnote}

Reporting Checklist: The authors have completed the STROBE reporting checklist. Available at http://dx.doi. org/10.21037/apm-21-791

Data Sharing Statement: Available at http://dx.doi. org/10.21037/apm-21-791

Conflicts of Interest: All authors have completed the ICMJE uniform disclosure form (available at http://dx.doi. org/10.21037/apm-21-791). All authors declare that this study was supported by The National Natural Science Foundation of China (Grant No. 81700581), Project of Science and Technology Department of Sichuan Province 
(Grant No. 2019YFH0069), Chengdu Medical Research Project (Grant No. 2020208), and the Sichuan Provincial People's Hospital Clinical Research and Transformation Fund (2018LY12).

Ethical Statement: The authors are accountable for all aspects of the work in ensuring that questions related to the accuracy or integrity of any part of the work are appropriately investigated and resolved. The study protocol was approved by the ethics committee of Sichuan Provincial People's Hospital. All participants provided written informed consent prior to data collection. All procedures performed in this study involving human participants were in accordance with the Declaration of Helsinki (as revised in 2013).

Open Access Statement: This is an Open Access article distributed in accordance with the Creative Commons Attribution-NonCommercial-NoDerivs 4.0 International License (CC BY-NC-ND 4.0), which permits the noncommercial replication and distribution of the article with the strict proviso that no changes or edits are made and the original work is properly cited (including links to both the formal publication through the relevant DOI and the license). See: https://creativecommons.org/licenses/by-nc-nd/4.0/.

\section{References}

1. Zhang L, Wang F, Wang L, et al. Prevalence of chronic kidney disease in China: a cross-sectional survey. Lancet 2012;379:815-22.

2. Data Source: Chinese National Renal Data System (CNRDS). Available online: http://hd.cnrds.net

3. SHERMAN R. A. Modifying the dialysis prescription to reduce intradialytic hypotension. Am J Kidney Dis 2001;38:S18-25

4. Palmer BF, Henrich WL. Recent advances in the prevention and management of intradialytic hypotension. J Am Soc Nephrol 2008;19:8-11.

5. Flythe JE, Inrig JK, Shafi T, et al. Association of

Cite this article as: Deng F, Di W, Ma Y, Li Y, Liao T, Mou J, Hong D, Wang W, Chen J, Jiang P, Li G, Mao J, Feng J. The relationship between prescription of ultrafiltration and intradialytic hypotension in Chinese hemodialysis patients. Ann Palliat Med 2021;10(5):5316-5321. doi: 10.21037/apm-21-791 intradialytic blood pressure variability with increased allcause and cardiovascular mortality in patients treated with long-term hemodialysis. Am J Kidney Dis 2013;61:966-74.

6. Nesrallah GE, Suri RS, Guyatt G, et al. Biofeedback dialysis for hypotension and hypervolemia: a systematic review and meta-analysis. Nephrol Dial Transplant 2013;28:182-91.

7. Chang TI, Paik J, Greene T, et al. Intradialytic hypotension and vascular access thrombosis. J Am Soc Nephrol 2011;22:1526-33.

8. Sands JJ, Usvyat LA, Sullivan T, et al. Intradialytic hypotension: frequency, sources of variation and correlation with clinical outcome. Hemodial Int 2014;18:415-22.

9. Stefánsson BV, Brunelli SM, Cabrera C, et al. Intradialytic hypotension and risk of cardiovascular disease. Clin J Am Soc Nephrol 2014;9:2124-32.

10. Bossola M, Laudisio A, Antocicco M, et al. Intradialytic hypotension is associated with dialytic age in patients on chronic hemodialysis. Ren Fail 2013;35:1260-3.

11. Rocha A, Sousa C, Teles P, et al. Frequency of intradialytic hypotensive episodes: old problem, new insights. J Am Soc Hypertens 2015;9:763-8.

12. Flythe JE, Kunaparaju S, Dinesh K, et al. Factors associated with intradialytic systolic blood pressure variability. Am J Kidney Dis 2012;59:409-18.

13. Mc Causland FR, Brunelli SM, Waikar SS. Dialysis dose and intradialytic hypotension: results from the HEMO study. Am J Nephrol 2013;38:388-96.

14. Depner TA. Assessing adequacy of hemodialysis: urea modeling. Kidney Int 1994;45:1522-35.

15. Trivedi HS, Kukla A, Prowant B, et al. A study of the extracorporeal rate of blood flow and blood pressure during hemodialysis. Hemodial Int 2007;11:424-9.

16. Schytz PA, Mace ML, Soja AM, et al. Impact of extracorporeal blood flow rate on blood pressure, pulse rate and cardiac output during haemodialysis. Nephrol Dial Transplant 2015;30:2075-9.

(English Language Editor: J. Teoh) 